\title{
The usefulness of growth hormone treatment for psychological status in young adult survivors of childhood leukaemia: an open-label study
}

\author{
Jaap Huisman*1, Eline J Aukema1,5, Jan Berend Deijen², Silvia CCM van \\ Coeverden ${ }^{3,6}$, Gertjan JL Kaspers ${ }^{3}$, Heleen JH van der $\mathrm{Pal}^{4}$ and \\ Henriette A Delemarre-van de $\mathrm{Waal}^{3}$
}

\begin{abstract}
Address: ${ }^{1}$ Department of Medical Psychology, VU University Medical Center, P.O. Box 7057, 1007 MB Amsterdam, The Netherlands, ${ }^{2}$ Department of Clinical Neuropsychology, VU University, van der Boechorststraat 1, 1081 BT, Amsterdam, The Netherlands, ${ }^{3}$ Department of Pediatrics, VU University Medical Center, P.O. Box 7057, 1007 MB Amsterdam, The Netherlands, ${ }^{4}$ Department of Medical Oncology, Academic Medical Center, P.O. Box 22660, 1100 DD Amsterdam, The Netherlands, ${ }^{5}$ Psychosocial Department, Emma Children's Hospital/Academic Medical Center, P.O. Box 22660, 1100 DD Amsterdam, The Netherlands and ' ${ }^{6}$ Department of Public and Occupational Health, EMGO-institute, van der Boechorststraat 7, 1081 BT Amsterdam, The Netherlands
\end{abstract}

Email: Jaap Huisman* - drj.huisman@vumc.nl; Eline J Aukema - e.j.aukema@amc.uva.nl; Jan Berend Deijen - jb.deijen@psy.vu.nl; Silvia CCM van Coeverden - S.vanCoeverden@vumc.nl; Gertjan JL Kaspers - GJL.Kaspers@vumc.nl; Heleen JH van der Pal - H.J.vanderPal@amc.uva.nl; Henriette A Delemarre-van de Waal - H.Delemarre@vumc.nl

* Corresponding author

Published: 20 June 2008

BMC Pediatrics 2008, 8:25 doi:10.1 I86/147|-243|-8-25
Received: 4 December 2007

Accepted: 20 June 2008

This article is available from: http://www.biomedcentral.com/I47I-243I/8/25

(C) 2008 Huisman et al; licensee BioMed Central Ltd.

This is an Open Access article distributed under the terms of the Creative Commons Attribution License (http://creativecommons.org/licenses/by/2.0), which permits unrestricted use, distribution, and reproduction in any medium, provided the original work is properly cited.

\begin{abstract}
Background: To reduce the risk of brain damage children with acute lymphoblastic leukaemia (ALL) are nowadays mainly treated with intrathecal chemotherapy (ITC) instead of central nervous system (CNS) radiation therapy (CRT) to prevent CNS relapse. However, chemotherapy may also lead to cognitive deficits. As growth hormone deficiency (GHD) or impaired growth hormone secretion are frequently found in ALL patients treated with cranial radiation therapy and/or chemotherapy, we hypothesized that $\mathrm{GH}$ therapy may reduce cognitive deficits in these patients.

Methods: Twenty young adult survivors of childhood ALL with reduced bone mineral density $(<-$ I SD) and/or low IGF-I SD-scores (<-I SD) were included in the study. A final group of I 3 patients ( 9 males and 4 females), mean age $23.7 \pm 2.9$ years (range $20-29.7$ ) completed a 2 -year treatment with $\mathrm{GH}$.

$\mathrm{IQ}$ and neuropsychological performance were assessed at pre-treatment (TI) and after one (T2) and two (T3) years. ANOVA was performed with assessment at TI, T2 and T3 as repeated measurements factor. Relations between test score changes and changes of IGF-I levels were determined by calculating the Pearson correlation coefficient.

Results: Scores on the cognitive tests were in the normal range. Verbal short- and long-term memory performance decreased between $\mathrm{TI}$ and $\mathrm{T} 2$, and increased between $\mathrm{T} 2$ and $\mathrm{T} 3$. Performance at T3 was not significantly different from that at TI. Performance for sustained attention improved from $\mathrm{TI}$ to $\mathrm{T} 2$ and from $\mathrm{TI}$ to $\mathrm{T} 3$. Visual-spatial memory was improved after one year of GH treatment. A significant positive correlation was found for $\Delta$ IGF-I (T2-TI) with difference scores of visual-spatial memory (T2-TI and T3-TI), indicating that IGF-I increase after
\end{abstract}


one year of $\mathrm{GH}$ treatment is associated with increase in cognitive-perceptual performance at month 12 and 24.

Conclusion: Since the level of intellectual functioning of our patient cohort was in the normal range the present finding that $\mathrm{GH}$ treatment has negative effects on verbal memory and positive on attention and visual-spatial memory warrants similar studies in other groups of ALL survivors. Also, a lower dose of $\mathrm{GH}$ should be determined inducing as much IGF as needed to improve verbal as well as visual cognitive functions. The present findings indicate that more knowledge is needed before GH treatment may be recommended to enhance cognitive functions in ALL survivors.

\section{Background}

In the last decades the prognosis of children with acute lymphoblastic leukaemia (ALL) has improved dramatically and long-term survival rates up to $80 \%$ have been reported [1]. Along with this development much research has been directed at identifying the psychological effects of treatment for ALL in childhood, in particular the neuropsychological sequelae. Prophylactic intrathecal (IT) chemotherapy (ITC) has replaced CNS radiation therapy (CRT), as research revealed cognitive deterioration and deficits associated with such CRT $[2,3]$. However, negative effects of high-dose IT chemotherapy regimens have been reported. For instance, children with ALL who had received chemotherapy for 3 years were more impaired on neurocognitive tasks involving right hemisphere simultaneous processing than their healthy siblings and than ALL children diagnosed recently or receiving chemotherapy for one year [4]. From a study by Copeland et al. [5] the cognitive side effects of IT chemotherapy appeared to be slightly more apparent 5 to 11 years after diagnosis than at 3-year follow-up. Since the differences were not clinically meaningful, the effects of chemotherapy in the absence of CRT were concluded to be slight. This is in accordance with the finding that the intellectual performance of survivors of paediatric ALL (age 6.8-33.7 years) treated with chemotherapy was normal [6]. In contrast, from thirty three reviewed studies on the long-term consequences of CNS chemotherapy in ALL survivors approximately two thirds document a decline in cognitive abilities [7]. For instance, patients treated with chemotherapy showed worse memory and fine-motor functions, but not school level, than their siblings [8]. Recently, behavior problems but also poor school performance was found in children with ALL attending primary school compared with same-age peers, although the rate of utilization of special education services was low. Thus, treatment for childhood ALL with chemotherapy may be associated with subtle but significant behavioral and educational problems [9].

Indeed, as with CRT, there is evidence that systemic CNSdirected and IT chemotherapy may lead to brain abnormalities, such as decreased cerebral perfusion, slower resting electroencephalogram frequencies, white matter changes and enlargement of the ventricles and cortical sulci [7].

From the studies cited above it may be concluded that cognitive impairment may be present in children with ALL who have been treated with CRT or IT chemotherapy. Therefore, a number of approaches to remediate these deficits, such as cognitive remediation, pharmacology and ecological alterations in the classroom, have been proposed. With respect to pharmacotherapy, in particular the use of phenylphenidate seems to be encouraging for ALL survivors with learning and attention problems [10].

Another pharmacological approach may be the use of growth hormone $(\mathrm{GH})$ treatment. Growth hormone deficiency (GHD) or impaired GH secretion are frequently found late effects in patients treated with cranial radiation therapy and/or chemotherapy for childhood ALL [11-13].

Cognitive functioning and IQ appears subnormal in patients with GHD, as patients complain of lapses of attention, difficulty in concentrating, and forgetfulness. Moreover, their IQ score and educational level appear to be positively related to the concentration of insulin-like growth factor I (IGF-I), a serum marker for GH status, suggesting that subnormal cognitive performance is specifically related to GHD $[14,15]$. The association between cognitive functions and GH may be explained by the presence of many binding sites of GH and IGF-I in the hippocampus, a brain structure that is important for learning and memory functions [16]. Some studies suggest that GH therapy can have beneficial effects for the cognitive functioning of GH deficient adults, in particular memory function [17] and attention [18,19]. The psychological effects of GH therapy in a group of survivors of childhood cancer have been investigated only once before. However, aim of that study was to establish health related Quality of Life of survivors (most of them ALL), whether or not with GHD requiring $\mathrm{GH}$ treatment, while effects on neurocognitive functioning were not studied [20].

The present study is the first that evaluated the effects of GH on neurocognitive functioning of ALL survivors with a low bone mass and/or low IGF-I levels. We hypothe- 
sized that GH therapy would show positive effects on neurocognitive functioning, in particular attention and memory, in adult survivors of paediatric ALL.

\section{Methods \\ Patients}

Young adult survivors of childhood leukaemia with reduced bone mineral density (<-1 SD) and/or low IGF-I SD-scores ( $<-1 \mathrm{SD})$ were selected from a group of patients, treated for ALL between 1972 and 1990 at the paediatric departments of the VU University Medical Center and the Academic Medical Center in Amsterdam. The inclusion criteria were chosen like this because originally the study was designed to see whether bone mineral density would improve after GH intervention. Twenty out of 56 (36\%) childhood ALL survivors over 20 years of age were included according to these criteria. These patients had never been tested for GHD and as a consequence were never treated with GH before.

Treatment of ALL had been given according to different treatment protocols. In general, treatment consisted of an intensive induction period, followed by a 2 to 3 year maintenance period. All patients received systemic cytostatic treatment, including corticosteroids, vincristine, methotrexate and 6-mercaptopurine and sometimes other cytostatic drugs were added. In addition, 17 patients out of the 20 included received prophylactic cranial irradiation in doses varying from 2000 to 2500 cGy. Three patients received high-dose methotrexate and intrathecal chemotherapy (methrotrexate, cytosine-arabinoside, prednison) instead of cranial irradiation to prevent central nervous system relapse.

ALL survivors were reviewed for age at diagnosis, treatment protocol, dose of irradiation, and time elapsed since irradiation. After an overnight fast a physical examination was performed and a blood sample was taken to assess IGF-I serum values. Blood samples were taken and IGF-I determined at baseline and at month 3, 6, 9, 12, 15, 18, 21 and 24. Mean IGF-I z-scores are shown in Fig. 1. Serum IGF-I was measured (in duplicate) after extraction by immunoradiometric assay (DSL, Webster, Texas USA). In addition, a provocative GH test was performed after overnight fast in all participants, using growth hormone releasing hormone in a dose of $100 \mu \mathrm{g}$ intravenously (GHRH-test). To assess GH levels, blood samples were drawn at $-15,0,10,20,30,45,60$ and 90 minutes. The subjects rested during the test.

At another morning the insulin tolerance test (ITT) was carried out in the ALL survivors. Intravenous soluble insulin (Velosulin) was administered in a dose of 0.1 IE insulin/kg body weight. To assess GH levels, blood samples were taken at $-15,0,10,20,30,45,60$ and 90 minutes.

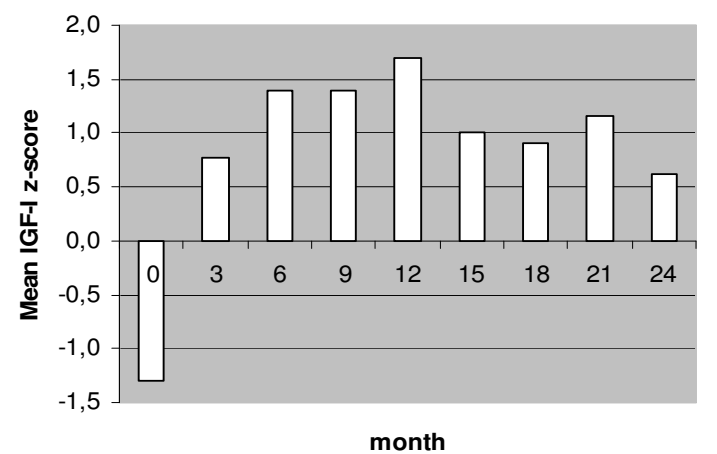

Figure I

Mean IGF-I Z-score at baseline (TI) and after 3, 6, 9, I 2 (T2), I 5, I 8, 2 I and 24 (T3) months of GH therapy.

During the ITT all subjects achieved blood glucose levels of $<2.0 \mathrm{mmol} / \mathrm{l}$ and there were clinical symptoms of hypoglycaemia as well. Blood glucose levels were measured every five minutes during the test.

The study protocol has been approved by the Medical Committee of Ethics on Human Research of the VU University Medical Centre and written informed consent was obtained from all patients.

\section{Procedure}

Diagnosis of $\mathrm{GH}$ deficiency

The diagnosis of GHD was based on a GH peak response less than $9.0 \mathrm{mU} / \mathrm{L}$ (3 microgram/l) in both provocative tests. Four patients showed a GH peak response below 9.0 $\mathrm{mU} / \mathrm{L}$ (3 microgram/l) for the ITT, but not for the GHRHtest; those patients were also considered growth hormone deficient. In this way, 13 out of 20 patients were GHD. Serum GH was measured in duplicate by immunometric assay (Sorin Biomedica, Saluggia Italy). The standards used were calibrated against the 1st IS 80/505 reference preparation.

\section{Growth hormone therapy}

Of the 20 ALL survivors with decreased BMD and/or IGFI, three patients did not start with GH, because one of them was diagnosed with anorexia nervosa and the other two refused treatment. The remaining 17 patients started with GH-therapy for a two-year period (10 males, 7 females; mean age $\pm \mathrm{SD}, 24.4 \pm 3.3$; range $20-31 \mathrm{yrs}$ ). Four patients stopped GH-therapy: two of them got pregnant within 3 months, one patient turned out to be growth hormone resistant and the other stopped for unknown reasons. A final group of thirteen participants ( 9 males and 4 females) completed the study. These participants were treated with growth hormone during 2 years. 
Four of them were not GHD (GH peak response in ITT between 11.0 and $70.0 \mathrm{mU} / \mathrm{l}$ ). Mean age at diagnosis was $7.0 \pm 4.3$ years (range $2.5-15.2$ years). Mean age at the start of the study was $23.7 \pm 2.9$ years (range $20-29.7$ years). Mean IGF-1 ( \pm SD) at the start of the study was $17.5 \pm 5.3$, and for IGF-Z-scores it was $-1.3 \pm 0.6 \mathrm{SD}$.

All patients used Humatrope subcutaneously with the Eli Lilly penfill system. The starting dose of GH was calculated as $0.1 \mathrm{mg}$ per square meters of body surface. Every 2 weeks, the dose was increased with $0.1 \mathrm{mg} / \mathrm{m} 2$, until IGFI rised above $0 \mathrm{SD}$. The GH dose that was needed to reach a level of IGF- 1 of +1 SD according to sex and age was very different per individual. In the first 3 months, patients were seen after 2, 4, 8 and 12 weeks, after that every 3 months.

\section{Assessment of (neuro)psychological functions}

Participants were tested 3 times: before the start of GH treatment (T1) and after 1 and 2 years of treatment (T2, T3). Assessments were carried out by a well-trained and qualified test-assistant. All tests employed were standardized and do have documented data about reliability and validity.

\section{Intelligence (assessment at TI)}

Patients' IQ score (Full scale (FS)IQ, Performal (P)IQ and Verbal (V)IQ) was assessed only at T1 with the Dutch version of the Wechsler Adult Intelligence Scale (W.A.I.S.) [21].

\section{Memory (assessments at TI, T2, T3)}

- 15 Word Test. Auditory verbal learning was tested in two conditions: immediate recall and recall after a 20-minute delay [22]. Two comparable versions were used (A at T1 and T3; B at T2) in order to minimize test-retest effects.

- Benton Visual Retention Test. In this test geometrical figures are presented and have to be recalled immediately by drawing. The test assesses visual memory, visual perception and visual-constructional capacities [23].

- Digit Span (subtest of WAIS). Digits are presented verbally and have to be recalled forward or reversed. Span of immediate verbal recall is tested.

- Rey-Osterrieth Complex Figure Test. After copy drawing of a complex geometrical figure subjects are asked to recall this drawing after 20 minutes. Visual-spatial memory is assessed [24]. To avoid test-retest effects we used two different figures: the Rey-Osterrieth figure at $\mathrm{T} 1$ and $\mathrm{T} 3$, and the Taylor figure at $\mathrm{T} 2$.
Attention (assessments at TI, T2, T3)

- Fepsy (Auditory Reaction Time) [25]. Auditory reaction time was assessed with the Auditory Reaction Time subtest, part of the Fepsy, a Dutch automated computerized test for cognitive functions. In this task the computer generates a $800 \mathrm{HZ}$ auditory stimulus and the subject has to produce as quick as possible a push-button response. Reaction time for the dominant and the non-dominant hands are measured.

-The Brickenkamp d2 test measuring sustained attention. In this test a person is asked to detect and encircle correct letter-quotation mark-combinations. Total score is the number of correctly encircled letters minus the number of incorrect responses [26].

Executive functions (assessments at TI, T2, T3)

- Trailmaking Test ( $A$ and $B$ ). Encircled numbers have to be connected consecutively by drawing lines (part A), or encircled letters and numbers have to be connected consecutively by alternating letters and numbers (part B). This test examines visual conceptual and visuomotor tracking [27].

\section{Data analysis}

Scores at T1 were compared to test norms (independent samples test). Statistical analysis comprised analyses of variance (ANOVA) and correlation analyses. To determine the effects of GH therapy ANOVA was used with assessment at T1, T2 and T3 as repeated measurements factor. If the factor assessment yielded significant main effects within-subjects contrasts were evaluated. To determine relations between test score changes and changes of IGF-I levels the Pearson correlation coefficient was calculated. Significance level was defined as $p \leq .05$ (one-tailed). Data were analysed using the SPSS version 11 software package (SPSS inc., Chicago, USA).

\section{Results}

Table 1 presents test scores prior to the start of GH treatment. The level of intellectual functioning (FSIQ, VIQ, PIQ) of the group survivors was high average. Memory tasks all resulted in test scores in the normal range of the population norms. Visual construction and visual motor tracking scores also were average in comparison to test norms. Sustained attention scores and auditory reaction time (dominant and non-dominant hand) were in the normal range as well.

Changes in IGF-I Z-score levels across the treatment period are presented in Fig. 1. Levels at T2 and T3 were significantly higher than at baseline $(F(1,12)=39.1, p \leq$ $.0005, \eta^{2}=0.76$ and $F(1,24)=29.5, p \leq .0005, \eta^{2}=0.71$, respectively). A significant decrease in IGF-I was seen between T2 and T3 $\left(F(12,24)=4.6, p=0.05, \eta^{2}=0.28\right)$. 
Table I: Mean pre-treatment (TI) scores \pm SD of ALL survivors $(n=13)$

\begin{tabular}{lll}
\hline Full scale IQ & $112 \pm 12$ & \\
Verbal IQ & $110 \pm 13$ & \\
$\begin{array}{l}\text { Performal IQ } \\
\text { Verbal short term memory }\end{array}$ & $113 \pm 13$ & High average \\
$\begin{array}{l}\text { I5 Word Test (number correct) } \\
\text { Verbal long term memory }\end{array}$ & $45.8 \pm 3.8$ & Average \\
$\begin{array}{l}\text { I5 Word Test Recall (number correct) } \\
\text { Visual-spatial short term memory }\end{array}$ & $9.2 \pm 1.8$ & Average \\
$\begin{array}{l}\text { Benton (number correct) } \\
\text { Short tem memory }\end{array}$ & $8.2 \pm 1.8$ & Average \\
$\begin{array}{l}\text { Digit Span (WAIS) (number correct) } \\
\text { Visual-spatial construction ability }\end{array}$ & $7.8 \pm 3.6$ & High average \\
$\begin{array}{l}\text { Rey (copy) (number correct) } \\
\text { Rey (LTM)(number correct) } \\
\text { Auditory RT }\end{array}$ & $34.92 \pm 1.5$ & \\
$\begin{array}{l}\text { Fepsy dominant hand (ms) } \\
\text { Fepsy non-dominant hand (ms) }\end{array}$ & $22.8 \pm 6.3$ & Average \\
sustained attention & $242 \pm 54.6$ & \\
$\begin{array}{l}\text { D2 (number correct) } \\
\text { Visual scanning and tracking }\end{array}$ & $239 \pm 56.9$ & Average \\
$\begin{array}{l}\text { Trailmaking A (sec) } \\
\text { Trailmaking B (sec) }\end{array}$ & $378 \pm 88.7$ & Average \\
\hline
\end{tabular}

Mean scores at $\mathrm{TI} \pm \mathrm{SD}$; mean IQ $=100, \mathrm{SD}=15$; higher test scores indicate better performance except scores in time (Auditory RT and Visual Scanning)

ANOVA showed significant differences between test sessions for Verbal short term memory (STM), Verbal long term memory (LTM), Visual-spatial long term memory and Sustained attention (see Table 2).

With respect to verbal short- and long-term memory a significant decrease in performance is seen between $\mathrm{T} 1$ and $\mathrm{T} 2$, followed by an increase between T2 and T3. The performance on verbal STM and verbal LTM is not significantly different between T1 and T3. As is clearly depicted in Fig. 2, the increase in IGF-I between T1 and T2 is accompanied by a decrease in short-term memory performance, while the decrease in IGF-I between T2 and T3 is accompanied by an increase in short-term memory scores. Exactly the same association with IGF-I was found for long-term memory.
Results for visual-spatial long term memory show an increase between $\mathrm{T} 1$ and $\mathrm{T} 2$ followed by a decrease between T2 and T3 (see table 2). The performance for visual-spatial long term memory at T3 only tends to be higher than at T1 $(p=0.07)$. An increase in performance for sustained attention is seen between T1 and T2. In addition, the performance at T3 is significantly better than at T1 $(p<0.002$, see table 2$)$.

As IGF-I levels were higher at T2 and T3 than at baseline we calculated the correlation between T2-T1 and T3-T1 difference scores of IGF-I and cognitive test scores. Significant positive correlations were only found of $\Delta$ IGF-I (T2T1) with $\Delta$ visual-spatial long term memory (T2-T1) and with $\Delta$ visual-spatial long term memory (T3-T1) $(r=0.58$, $p=0.02$ and $r=0.53, p=0.04$, respectively). This means that IGF-I increase after one year of GH treatment is associated with increase in cognitive performance at month 12 and 24 relative to baseline. There was no correlation between $\Delta$ IGF-I (T3-T1) with $\Delta$ visual-spatial long term memory (T3-T1).

\section{Discussion}

In literature the neurocognitive late effects of the treatment of childhood leukaemia have been studied extensively. Deleterious effects of treatment regimens including cranial radiation in particular have been documented. Results of studies on the effects of prophylactic CNS treatment with high-dose and intrathecal chemotherapy however show that such therapy also may have deleterious effects. Key question in the present study was whether GH treatment improves neurocognitive functioning of adult survivors of childhood leukemia. We hypothesized positive effects of GH treatment, in particular improvement of attention and memory, based on effect studies in growth hormone deficient adults [17-19].

In the present study a group of 13 young adult survivors of childhood leukaemia was assessed neuropsychologically before and during GH therapy installed because of decreased BMD and/or too low IGF-I. Most persons (10 of 13) had been treated with regimens including prophylactic cranial irradiation. Many years after treatment (mean time since diagnosis approximately 15 years) these survi-

Table 2: Mean cognitive scores \pm SD and significance levels of factor Session

\begin{tabular}{|c|c|c|c|c|c|c|}
\hline & \multicolumn{3}{|c|}{ Session } & \multicolumn{3}{|c|}{ p-value (session) } \\
\hline & 1 & 2 & 3 & $\mathrm{I}-2$ & $2-3$ & $1-3$ \\
\hline Verbal short-term memory & $46 \pm 3.8$ & $40 \pm 9.5$ & $49 \pm 6.9$ & 0.03 & 0.000 & 0.14 \\
\hline Verbal long-term memory & $9.2 \pm 1.8$ & $6.4 \pm 3.0$ & $9.6 \pm 2.8$ & 0.008 & 0.000 & 0.41 \\
\hline Visual-spatial long-term memory & $22.8 \pm 6.3$ & $28.2 \pm 4.5$ & $26.0 \pm 3.9$ & 0.004 & 0.05 & 0.07 \\
\hline Sustained attention & $378 \pm 89$ & $421 \pm 100$ & $435 \pm 102$ & 0.02 & 0.45 & $<0.002$ \\
\hline
\end{tabular}

Higher test scores indicate better performance 


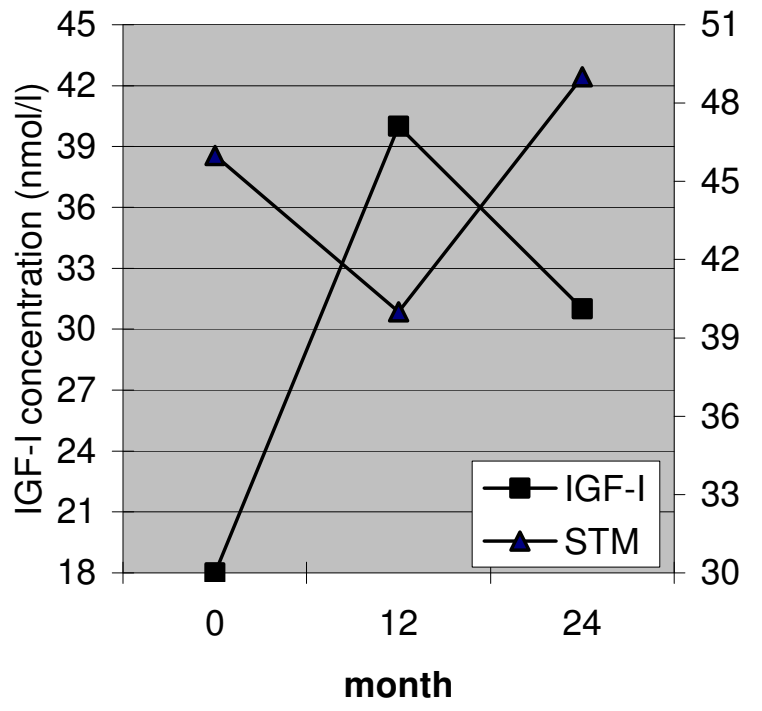

Figure 2

Mean IGF-I levels (nmol/I) and Verbal short term memory scores (I 5 Word Test - immediate recall) prior to start of $\mathrm{GH}$ treatment and after 12 and 24 months.

vors showed test scores on a variety of neuropsychological tasks in the normal range. Mean level of intellectual functioning as determined by IQ tests was even high average. This level is higher than expected, since most participants received prophylactic cranial irradiation as part of their ALL-treatments. The same holds for the test scores relating to specific neurocognitive functions we measured. Among others deficits in memory, visuospatial/motor skills and attention have been reported consistently in literature $[7,28,29]$.

Although all available survivors meeting the growth and age criteria were invited to participate in this study and a high percentage (85\%) agreed to do so, our small study group may be a quite selected sample not reflecting the cognitive functioning of the average population of ALL survivors. Because we have no information of pre-morbid functioning of these patients, and since our study lacks a control group, we can not exclude general or individual detrimental effects of ALL-treatment. Further, the observed high average IQ scores may be skewed due to the Flynn effect, that is Dutch test norms of the WAIS were published in 1970 and gains of IQ-points over time can add up from 2 to 3 points per decade [30]. However, if we adjust the mean high average IQ scores in the present patient group for the Flynn effect, these scores would be still in the normal range. This is in accordance with Von der Weid et al. [6] who described normal cognitive outcome in adolescent and young adult survivors of ALL.
These survivors, however, were treated with chemotherapy alone. Since literature suggests different neurocognitive outcome in children with ALL treated with or without CNS irradiation and in view of our controversial findings concerning scores of intellectual and specific neuropsychological tests in adult survivors, the need for further investigation of the very long term neurocognitive effects of treatment of childhood ALL, with or without cranial irradiation, in adult survivors is underlined.

In the present study IGF-I levels were used as expression of neuroendocrine effects of growth hormone therapy and were related to testscores. Mean IGF-I level increased in the first year of treatment, decreased in the second year, but remained higher than at pre-treatment. After one year of GH treatment scores on tests measuring verbal shortand long-term memory decreased as IGF-I levels increased. After the second year of treatment the shortand long-term memory scores increased to the levels at T1 as IGF-I decreased. Thus, with respect to memory, an increase in IGF-I in the first year of treatment seems to be associated with impaired memory performance, which impairment seems to be counteracted by a reduced IGF-I level. In addition, sustained attention was significantly improved after the first and second treatment year, as IGFI was highly increased. The absence of a difference in response on sustained attention between session 2 and 3 may be associated with the reduced IGF-I levels after one year of GH treatment. All in all, the GH-induced IGF-I increase in the first treatment year seems to impair specific (verbal) memory functions, which conclusion may be inferred from the observation that memory impairment is halted when IGF-I levels are lowered. It may well be true that the IGF-I increase in the fist year is too high to improve verbal memory functions which even results in an opposite effect, that is impairment of verbal memory. An other possible explanation could be that IGF-1 sensitivity of the brain is altered by prophylactic CRT.

Positive effects found in the present study concerns visualspatial long term memory and sustained attention, which functions are improved after one year of treatment and respectively tend to be or are still improved after the second treatment year. Correlation analysis indicated that the IGF-I increase in the first treatment year is associated with an improvement of visual-spatial long term memory at month 12 and 24 relative to baseline. No relation was found between the increase in IGF-I during two years of treatment and changes in visual-spatial long term memory. As perceptual skills play a role in both spatial longterm memory and sustained attention, this result suggests that especially cognitive perceptual performance after one and two years of GH treatment are related to the IGF-I increase in the first year. Indeed, a relationship between IGF-I levels and perceptual functions has been reported by 
Aleman et al. [31]. In healthy male subjects (mean age of 69 years) higher IGF-1 levels were associated to better scores on the Digit Symbol Substitution test (W.A.I.S.), which test measures perceptual-motor functions and visual-spatial memory. As visual-spatial memory plays an important role in the performance on the Rey complex figure test it may well be true that a substantial increase in IGF-I may enhance visual-spatial (memory) functions.

We conclude that the present results show some beneficial effects of GH treatment on visual-spatial (long term) memory functions and attention in ALL survivors, while verbal memory functions are negatively affected.

\section{Conclusion}

This study is the first presenting data about the neurocognitive functioning of adult survivors of childhood leukaemia in relation to GH treatment. Since most participants were treated with regimens including prophylactic cranial irradiation, the finding that these survivors showed at least an average level of intellectual functioning and neuropsychological test scores in the normal range is unexpected and asks for further research. The present finding that GH treatment has negative effects on verbal memory and positive effects on visual-spatial memory and attention warrants similar studies in other groups of ALL survivors. However, because of the unexpected high level of cognitive functioning and low levels of IGF-1 of our sample care is needed to generalize our results to the ALL population as a whole. In addition, the average baseline levels of cognitive functioning in our study group and positive effects of GH treatment on two particular neuropsychological tests while effects on other tests were absent or negative, questions clinical relevance. Further, because of these different effects of GH treatment on the neuropsychological tests observed positive effects may result from chance. In addition to the possibility that GH treatment may help ALL survivors with worse intellectual functioning, a lower dose of GH may be more effective as an increase too high of IGF-I may adversely affect verbal memory performance. However, it will be difficult to precisely determine the effective dose to improve verbal as well as visual cognitive functions, because a dose too low may in turn not affect visual memory and attention.

All in all, conclusions from studies in younger groups of survivors on neurocognitive toxicity of leukemia treatment, may not hold in all cases of young adult survivors. In addition, as has been described before in growth hormone deficient adults [17-19], the results of the present study suggest that relationships between GH therapy and aspects of neuropsychological functioning are strongly dependent on IGF-I levels. For theoretical and clinical reasons replication of this study and further research into these complex relationships is strongly recommended.
Based on the present results, we conclude that more knowledge is needed before $\mathrm{GH}$ treatment to enhance cognitive functions in ALL survivors may be recommended.

\section{Competing interests}

The authors declare that they have no competing interests.

\section{Authors' contributions}

$\mathrm{JH}$ and EJA made substantial contributions to conception and design, acquisition, analysis, and interpretation of data and writing the manuscript. JBD was involved in analysis, interpretation of data and writing the manuscript. SCCMvC, GJLK, HJHvdP and HAD-vdW made substantial contributions to conception and design of the study. All authors read and approved the final manuscript.

\section{Acknowledgements}

The authors wish to thank the participants for their kind participation in this study and Lilly Nederland BV for their financial support. We also thank Mrs. M.J. Koole for administering the neuropsychological tests.

This study was carried out under the auspices of the Institute for Clinical and Experimental Neurosciences - ICEN, VU University Medical Center, Amsterdam

\section{References}

I. Pui $\mathrm{CH}$ : Acute lymphoblastic leukemia in children. Curr Opin Oncol 2000, 12:3-12.

2. Moore BD: Neurocognitive outcomes in survivors of childhood cancer. J Pediatr Psychol 2005, 30:5I-63.

3. Mulhern SK, Palmer SL: Neurocognitive late effects in pediatric cancer. Curr Probl Cancer 2003, 27: 177-97.

4. Brown RT, Madan-Swain A, Pais R, Lambert RG, Sexson S, Ragab A: Cognitive status of children treated with central nervous system prophylactic chemotherapy for acute lymphocytic leukemia. Arch Clin Neuropsychol I992, 7:48I-97.

5. Copeland DR, Moore BD, Francis DJ, Jaffe N, Culbert SJ: Neuropsychologic effects of chemotherapy on children with cancer: a longitudinal study. J Clin Oncol 1996, 14:2826-35.

6. Von der Weid N, Mosimann I, Hirt A, Imbach P, Nenadov Beck M, Wacker P, Caflisch U, Niggli F, Feldges A, Wagner HP: Intellectual outcome in children and adolescents with acute lymphoblastic leukaemia treated with chemotherapy alone: age- and sex-related differences. Eur J Cancer 2003, 39:359-65.

7. Moleski M: Neuropsychological, neuroanatomical, and neurophysiological consequences of CNS chemotherapy for acute lymphoblastic leukemia. Arch Clin Neuropsychol 2000, 15:603-30.

8. Kingma A, van Dommelen RI, Mooyaart EL, Wilmink JT, Deelman BG, Kamps WA: Slight cognitive impairment and magnetic resonance imaging abnormalities but normal school levels in children treated for acute lymphoblastic leukemia with chemotherapy only. J Pediatr 200I, 139:4I3-20.

9. Buizer AI, de Sonneville LM, Heuvel-Eibrink MM van den, Veerman AJ: Behavioral and educational limitations after chemotherapy for childhood acute lymphoblastic leukemia or Wilms tumor. Cancer 2006, 106:2067-75.

10. Butler RW, Mulhern RK: Neurocognitive interventions for children and adolescents surviving cancer. J Pediatr Psychol 2005, 30:65-78.

II. Haddy TB, Mosher RB, Nunez SB, Reaman GH: Growth hormone deficiency after chemotherapy for acute lymphoblastic leukemia in children who have not received cranial radiation. Pediatr Blood Cancer 2006, 46:258-6I.

12. Gurney JG, Ness KK, Sibley SD, O'Leary M, Dengel DR, Lee JM, Youngren NM, Glasser SP, Baker KS: Metabolic syndrome and 
growth hormone deficiency in adult survivors of childhood acute lymphoblastic leukemia. Cancer 2006, 107:1303-I2.

13. Link K, Moell C, Osterberg K, Persson R, Orbaek P, Garwicz S, Cavallin-Stahl E, Erfurth EM: Adult survivors of childhood acute lymphoblastic leukaemia with GH deficiency have normal selfrated quality of life but impaired neuropsychological performance 20 years after cranial irradiation. Clin Endocrinol (Oxf) 2006, 65:617-25.

14. Deijen JB, de Boer H, Blok GJ, Veen EA van der: Cognitive impairments and mood disturbances in growth hormone deficient men. Psychoneuroendocrinology 1996, 21 :313-22.

15. Rosen T, Wiren L, Wilhelmsen L, Wiklund I, Bengtsson BA: Decreased psychological well-being in adult patients with growth hormone deficiency. Clin Endocrinol (Oxf) 1994, 40: I I I-6.

16. van Dam PS, Aleman A, de Vries WR, Deijen JB, Veen EA van der, de Haan EA, Koppeschaar HP: Growth hormone, insulin-like growth factor I and cognitive function in adults. Growth Horm IGF Res 2000:569-73.

17. Deijen JB, de Boer H, Veen EA van der: Cognitive changes during growth hormone replacement in adult men. Psychoneuroendocrinology 1998, 23:45-55.

18. Oertel H, Schneider HJ, Stalla GK, Holsboer F, Zihl J: The effect of growth hormone substitution on cognitive performance in adult patients with hypopituitarism. Psychoneuroendocrinology 2004, 29:839-50.

19. Soares CN, Musolino NR, Cunha NM, Caires MA, Rosenthal MC, Camargo CP, Bronstein MD: Impact of recombinant human growth hormone (RH-GH) treatment on psychiatric, neuropsychological and clinical profiles of $\mathbf{G H}$ deficient adults. A placebo-controlled trial. Arq Neuropsiquiatr 1999, 57: I82-9.

20. Eiser C, Vance YH, Glaser A, Galvin H, Horne B, Picton S, Stoner A, Butler G: Growth hormone treatment and quality of life among survivors of childhood cancer. Horm Res 2005, 63:300-4.

21. Stinissen J, Willems PJ, Coetsier P, Hulsman WLL: WAIS, nederlandse bewerking. Lisse: Swets \& Zeitlinger B.V; 1970.

22. Saan RJ, Deelman BG: Nieuwe I5-Woorden Test A en B. In Handboek Neuropsychologische Diagnostiek Edited by: Bouma A, Mulder J, Lindeboom J. Lisse: Swets \& Zeitlinger B.V; 1998.

23. Steck PH: A revision of A. L. Benton's Visual Retention Test (BVRT) in two parallel forms. Arch Clin Neuropsychol 2005, 20:409-16.

24. Shin MS, Park SY, Park SR, Seol SH, Kwon JS: Clinical and empirical applications of the Rey-Osterrieth Complex Figure Test. Nat Protoc 2006, I:892-9.

25. Riecher-Rossler A, Gschwandtner U, Aston J, Borgwardt S, Drewe M, Fuhr P, Pfluger M, Radu W, Schindler Ch, Stieglitz RD: The Basel early-detection-of-psychosis (FEPSY)-study - design and preliminary results. Acta Psychiatr Scand 2007, I | 5: | | 4-25.

26. Brickenkamp R: Test d2. Aufmerksamkeitsbelastungtest. Handanweisung. Gottingen: Hochrefe; 1978.

27. Reitan RM, Wolfson D: Category Test and Trail Making Test as Measures of Frontal Lobe Functions. Clin Neuropsychol 1995, 9:50-6.

28. Langer T, Martus P, Ottensmeier H, Hertzberg H, Beck JD, Meier W: CNS late effects after ALL therapy in childhood. Part III: neuropsychological performance in long-term survivors of childhood ALL: impairments of concentration, attention, and memory. Med Pediatr Oncol 2002, 38:320-8.

29. Spiegler BJ, Kennedy K, Maze R, Greenberg ML, Weitzman S, Hitzler JK, Nathan PC: Comparison of long-term neurocognitive outcomes in young children with acute lymphoblastic leukaemia treated with cranial radiation or high-dose or very high-dose intravenous methotrexate. J Clin Oncol 2006, 24:3858-64.

30. Flynn JR: Massive gains in I4 nations: What IQ tests really measure. Psychol Bull 1987, 101:171-91.

31. Aleman A, Verhaar HJ, de Haan EH, de Vries WR, Samson MM, Drent ML, Veen EA van der, Koppeschaar HP: Insulin-like growth factorI and cognitive function in healthy older men. J Clin Endocrinol Metab 1999, 84:47|-5.

\section{Pre-publication history}

The pre-publication history for this paper can be accessed here: http://www.biomedcentral.com/1471-2431/8/25/prepub
Publish with Biomed Central and every scientist can read your work free of charge

"BioMed Central will be the most significant development for disseminating the results of biomedical research in our lifetime. "

Sir Paul Nurse, Cancer Research UK

Your research papers will be:

- available free of charge to the entire biomedical community

- peer reviewed and published immediately upon acceptance

- cited in PubMed and archived on PubMed Central

- yours - you keep the copyright

Submit your manuscript here:

http://www.biomedcentral.com/info/publishing_adv.asp
BioMedcentral 